\title{
REVISIÓN/REVIEW
}

\section{NECESIDAD DE LA ALFABETIZACIÓN INFORMACIONAL EN LA EDUCACIÓN SUPERIOR}

Isabel González Flores': Universidad de La Habana. Cuba.

iflores@uci.cu

\section{RESUMEN}

La presente investigación aborda la necesidad de la Alfabetización Informacional en las instituciones de enseñanza superior cubanas, sus antecedentes y aspectos teóricos relacionados con la Alfabetización Informacional en Cuba y el mundo. Se plantea que la arista que con mayor frecuencia ha sido tratada por la comunidad cubana es en el diseño de programas para su ejecución, sin embargo los mismos no han sido suficientes para que existe una cultura generalizada de las ALFIN por parte de nuestros estudiantes universitarios, los cuales realizan investigaciones bibliográficas para la realización de sus trabajos de diploma y de cualquier otro tipo.

PALABRAS CLAVE: Alfabetización informacional - Investigación bibliográfica Aprendizaje - Competencias - Educación Superior

\footnotetext{
1 Autor correspondiente:

Isabel González Flores: Centro de Gobierno Electrónico (CEGEL), Facultad 3 de la Universidad de las Ciencias Informáticas de La Habana. Cuba.

Correo: iflores@uci.cu
} 


\title{
NECESSITY OF INFORMATION LITERACY IN HIGHER EDUCATION
}

\begin{abstract}
This paper is about the need to information literacy in higher education institutions in Cuba, its history and theoretical aspects related to Information Literacy in Cuba and the world. We propose that the edge that most often has been treated by the Cuban community is in the design of programs to run, however they have not been sufficient for there is a pervasive culture of the IL by our college students, who carry out literature searches to perform their jobs diploma or otherwise.
\end{abstract}

KEY WORDS: Information literacy - Bibliographic research - Learning - Skills - Higher Education

\section{INTRODUCCIÓN}

El impacto de las Tecnologías de la Información y las Comunicaciones (TIC), la gestión del conocimiento y la tendencia a la globalización son elementos característicos de la sociedad actual. Las fronteras entre la información y lo usuarios van disminuyendo, obligando al ser humano adaptar las capacidades y conocimientos para no quedar ajeno al desarrollo exponencial de las TIC.

Como menciona Félix Benito Morales ${ }^{2}$ en su documento Cuestiones previas a un proyecto Alfabetización informacional (ALFIN), 2007: "es un nuevo proceso de selección, que algunos autores denominan darwinismo social, donde ya no dependemos de la fuerza para sobrevivir, sino de la formación; una formación cuyo fin último está ligado al desarrollo personal, a la empleabilidad y a la relación interpersonal". Ante esta situación se deben establecer entornos educativos diferentes, capaces de adaptarse a tales circunstancias.

La abundante información que circula en la actualidad muchas veces llega sin filtrar, dando lugar a interrogantes sobre su autenticidad, validez y fiabilidad, además de plantear nuevos retos a los individuos para evaluar, comprender y utilizar la información de manera legal y ética, surgiendo términos como ALFIN. Por lo general cuando se habla de ALFIN se vincula con el conjunto de destrezas relacionadas con la localización, manejo y utilización de la información de forma eficaz para diferentes finalidades, aplicables a cualquier campo y a cualquier momento de la vida. Se trata, además, de una competencia genérica que permite a las personas enfrentar la toma de decisiones, la solución de problemas o la investigación, permitiéndoles responsabilizarse de su formación personal y en aprendizaje permanente.

2 Doctor en Pedagogía de España. Junto con José Antonio Gómez ha escrito alguno de los textos fundamentales de la ALFIN en España. Defiende un tipo de enseñanza distinto al tradicional, vinculándolo al concepto de "aprender a aprender", con la "educación documental" como elemento transversal. 
Acertadamente el documento de las Normas sobre alfabetización en información del Council of Australian University Librarians indica: " $\mathrm{La}$ ingente abundancia de información y de tecnología no creará por sí misma una ciudadanía más informada sin una comprensión y capacidad complementaria para usar la información eficazmente".

Desde la antesala de la sociedad del conocimiento, se ha mencionado por qué es necesaria una ALFIN, sin embargo existen numerosos estudiantes que arriban al último año de la carrera con escasas habilidades en la búsqueda, selección, evaluación, uso y diseminación de la información. En diferentes universidades del país se han desarrollado trabajos y cursos de postgrado relacionados con el tema, ejemplo de ello es la tesis de maestría de Liuris Rodríguez Castilla ${ }^{3}$, profesora de la Universidad de las Ciencias Informáticas, que propone un programa de ALFIN orientado a los proyectos productivos de la UCI.

La arista de la ALFN que con mayor frecuencia ha sido tratada por la comunidad cubana es el diseño de programas para su ejecución, sin embargo las experiencias que existen no han sido suficientes para incorporar este indispensable elemento a la práctica efectiva y constante de los estudiantes, los cuales arriban al último año de la carrera con limitadas habilidades y conocimientos sobre el uso de la información, elemento de vital importancia para el desarrollo de sus trabajos de diploma.

Los estudiantes necesitan incrementar sus capacidades y habilidades, de manera que favorezca el auto-aprendizaje organizado y que acreciente su potencial creativo, antes de enfrentarse al desarrollo del trabajo de diploma. Los mismos se interesan por nuevas formas de enseñanza y de aprendizaje autónomo, además de poseer un conocimiento cercano a las tecnologías. A pesar de esto se observan dificultades al relacionarse con el gran cúmulo de información, así como durante la selección, evaluación, utilización y formas de transferir la información.

\section{METODOLOGÍA}

Para abordar el concepto de alfabetización se ha recurrido al método comparativo, pues nos permite establecer mediante la comparación las analogías y diferencias existentes entre los distintos objetos, fenómenos, procesos y sus propiedades en el ámbito de la Alfabetización Informacional. Gracias a ello se pueden alcanzar resultados derivados de estos análisis y apoyándonos en el método de generalización, podremos expresar las regularidades esenciales que caracterizan las relaciones entre los diferentes objetos, fenómenos, procesos o sus características y se expresa en la conceptualización, extensión y transferencia de los resultados al ámbito docente.

\footnotetext{
${ }^{3}$ Máster en Gestión de Proyectos Informáticos. División de Servicios Informativos y Comunicación Empresarial (DISICE). La Habana, Cuba.
} 


\section{LA ALFABETIZACIÓN INFORMACIONAL EN LA SOCIEDAD CONTEMPORÁNEA}

La ALFIN es un proceso de gran importancia en la sociedad actual, donde el conocimiento y la información son indispensables para desarrollo de las sociedades contemporáneas. El crecimiento exponencial del volumen de información y la renovación del conocimiento, disponible en múltiples formatos, plantea nuevos retos para evaluar, comprender y utilizar la información de manera legal y ética, dando lugar a interrogantes sobre su autenticidad, validez y fiabilidad.

Un aspecto importante de la sociedad actualidad es la necesidad de que cada persona obtenga e incremente destrezas transferibles y utilizables a lo largo de la vida. El Council of Australian University Librarians, en el 2002, afirma que la ALFIN abarca el aprendizaje, el pensamiento crítico y las habilidades de interpretación cruzando fronteras profesionales y potenciando a individuos.

La ALFIN se ha convertido en un punto de primer orden para diferentes organizaciones como la IFLA ${ }^{4}$ y la UNESCO ${ }^{5}$, evidenciándose, entre otras acciones, en la organización de los Coloquios sobre ALFIN, como el de Praga en el 2003 y el de Alejandría en el 2005, y la celebración en el 2008 de una Conferencia Internacional. IFLA, por su parte organizó dos seminarios previos a la Cumbre Mundial de la Sociedad de la Información en Ginebra (IFLA 2003) y Túnez (IFLA 2005) y junto a la UNESCO han aceptado el reto de liderar los desarrollos de una Agenda Internacional de ALFIN con vistas a su promoción y expansión en todos los países (Ruíz, 2009).

\subsection{Necesidad de la Alfabetización Informacional en la Enseñanza Superior.}

Las condiciones y tendencias del mundo actual obligan a las instituciones de enseñanza superior a adaptarse a los nuevos cambios, adoptando modelos de formación que auspicien el autoa-prendizaje organizado y el potencial creativo de sus futuros egresados.

En este sentido las universidades tienen el deber de formar profesionales de excelencia capaces de adquirir, compartir y transmitir conocimiento durante toda su vida, que no estén preparados únicamente para un tipo de trabajo; que puedan adaptarse a las nuevas circunstancias que impone una sociedad cambiante y que evoluciona con gran rapidez, que sean capaces de captar nuevas ideas y conocimientos, así como de evaluar de forma crítica, que se cuestionen las cosas y no puedan vivir en la incertidumbre.

El marco social actual demanda de individuos con conocimientos, habilidades y valores necesarios para interactuar eficazmente con la información, ya sea para el interés personal o profesional, y que sientan la necesidad de mantenerse actualizados

4IFLA: International Federation of Library Associations.

${ }^{5}$ UNESCO: United Nations Educational, Scientific and Cultural Organization. 
en cuanto a los desarrollos recientes de ámbito de trabajo e investigación. Precisamente la ALFIN se ocupa de ello, propiciando una alternativa para desarrollar el aprendizaje más allá del entorno formal del aula y apoyando a los individuos en su aprendizaje auto dirigido en todas las circunstancias de la vida; implica un espectro mucho más amplio que el uso de la información.

El logro de soltura en ALFIN exige comprender que esta gama de habilidades no es algo extraño al propio plan de estudios, sino que está entretejida en el contenido, estructura y secuencia del currículo. Esta integración curricular también ofrece muchas posibilidades de aumentar la influencia e impacto de métodos de aprendizaje centrados en el estudiante, tales como el aprendizaje basado en problemas, el aprendizaje basado en evidencias o el aprendizaje mediante investigación (Council of Australian University Librarians, 2002).

La ALFIN se ha convertido en una necesidad y una exigencia en la enseñanza universitaria. Muy pronto los empleados se cotizarán por sus conocimientos y por el valor añadido que puedan generar, por tanto el principal reto de los sistemas educativos será potenciar la adquisición, estructuración y organización del conocimiento, desarrollando el capital intelectual de los estudiantes y dotándolos de un amplio inventario de habilidades y competencias para la resolución de problemas (Ruíz, 2009).

\subsection{Concepto de Alfabetización en información.}

Diferentes términos han sido utilizados en la literatura relacionada con la ALFIN, los cuales suelen usarse como sinónimos:

- $\mathrm{AI}$

- ALFIN

- Alfabetización digital.

- Alfabetización informacional.

- Alfabetización en información.

- Alfabetización bibliotecaria.

- Alfabetización tecnológica.

$\bullet$

De la misma forma diferentes autores han conceptualizado la ALFIN:

- Bawden ${ }^{6}$ considera que el término fue utilizado por primera vez por Paul Zurkowski ${ }^{7}, 1974$, en un trabajo para la Comisión Nacional de Biblioteconomía y Documentación de Estados Unidos, en el cual la ALFIN se asoció al concepto de "Destrezas de Información" dentro de un entorno laboral y con la resolución de problemas(Bawden, 2002).

- En 1979 Taylor introdujo el término en la literatura sobre bibliotecas, a la vez que aumentaba su área de aplicación. Su definición incluía las siguientes premisas: "que la solución a muchos problemas (no a todos) puede facilitarse

\footnotetext{
${ }^{6}$ David Bawden: Department of Information Science, City University London.

7 Paul Zurkowski: Presidente de la Asociación de Industrias de la Información.
} 
mediante la adquisición de hechos informacionales adecuados; que el conocimiento de la variedad de recursos disponibles es un requisito para esta alfabetización; que el proceso de información, que es continuo, es tan importante como el proceso de información puntual, que es ocasional; y que existen unas estrategias de adquisición de información" (Bawden, 2002).

- En 1994 Doyle reduce el concepto definido por Taylor, 1979, en una oración más sucinta y con similares principios: "La ALFIN es la capacidad de acceder, evaluar y utilizar la información a partir de una variedad de fuentes"(Bawden, 2002).

- Bruce ${ }^{8}$ define la ALFIN como: "un conjunto de aptitudes para localizar, manejar y utilizar la información de forma eficaz para una gran variedad de necesidades"(Bruce, 2003).

- El Council of Australian University Librarians define la ALFIN como una capacidad de comprender y un conjunto de habilidades que capacitan a los individuos para "reconocer cuándo se necesita información y poseer la capacidad de localizar, evaluar y utilizar eficazmente la información requerida" (Council of Australian University Librarians, 2002).

- La American Library Association9 (ALA) caracteriza a la AI como "la capacidad de comprender un conjunto de habilidades que capaciten a los individuos para reconocer cuando necesitan información y poseen habilidad para localizarla, evaluarla y usarla eficientemente en el punto de la necesidad".

- Martí Lahera10 concibe la "cultura informacional como un conjunto de conocimientos, valores y hábitos que, complementados con el uso de prácticas y herramientas de gestión de información en el actuar diario, permite a los miembros de una organización o sociedad aportar los retos que se presentan en el cumplimiento de los objetivos y metas a nivel micro y macro social".

- Erika Castillo Sáez afirma que la ALFIN establece el marco para la comprensión, hallazgo, evaluación y utilización de la información; inicia, mantiene y amplía el aprendizaje continuo a lo largo de toda la vida por medio de competencias que pueden servirse de las tecnologías pero que, en última instancia, son independientes de ellas (Sáez, 2005).

En los conceptos estudiados se observa que los autores hacen énfasis en la capacidad de los individuos para identificar la necesidad de información, así como en la búsqueda, evaluación y utilización la información que fluye por medio de las tecnologías. Sin embargo la ALFIN es un proceso mucho más complejo, que implica la transformación de la propia sociedad y es parte integral de la interacción social en la que el individuo desarrolla su personalidad.

A partir del análisis de los conceptos mencionados se concluye que "la ALFIN es el conjunto de habilidades que sea capaz de desarrollar un individuo para el uso de la

\footnotetext{
${ }^{8}$ Christine Susan Bruce: Queensland University of Technology, Brisbane, Australia.

${ }^{9}$ Asociación de Bibliotecarios Americanos.

10 Máster en Bibliotecología y Ciencia de la Información por la Facultad de Comunicación de la Universidad de La Habana en el año 2006. Profesor Instructor del Departamento de Bibliotecología y Ciencia de la Información.
} 
información, ya sea en formato duro o digital; resaltando como habilidades fundamentales la definición de necesidades de información, su localización, evaluación y organización y que todas en su conjunto, tributen a la generación de nuevos conocimientos, que al comunicarlos de manera ética, se convierta en nueva información", como expresara la MSc. Liuris Rodríguez.

\subsection{La Alfabetización Informacional como competencia transversal.}

Las competencias se definen como la capacidad de poner en marcha de manera integrada aquellos conocimientos adquiridos y rasgos de personalidad que permiten resolver situaciones diversas en la vida profesional (Pinto Molina, y otros, 2006).

El aprendizaje por competencias tiene mucho que ver con la educación general de los individuos, incluye conocimientos teóricos, habilidades prácticas o aplicativas, al igual que actitudes personales. Contribuye a la formación de habilidades y capacidades, ya sea para apropiarse del conocimiento como para saber utilizarlo en diferentes contextos de la realidad e implica que el estudiante sea el protagonista de su propio proceso de aprendizaje.

Entre los tipos de competencias se encuentran las:

- Competencias genéricas o transversales.

- Competencias básicas.

- Competencias específicas.

La ALFIN la competencia clave que posibilita el aprendizaje, permitiendo saber cuándo y por qué necesita información, dónde encontrarla y cómo evaluarla, utilizarla y comunicarla de manera ética, efectiva y eficiente. Pretende formar al individuo en el dominio de las competencias genéricas o transversales, transferibles a diferentes funciones y tareas, que capacitan y habilitan al estudiante para integrarse con éxito en la vida laboral y social. No son exclusivas de ninguna especialidad profesional, sino que se pueden aplicar a una variedad de áreas de materias y situaciones.

\subsection{Estándares de Alfabetización Informacional.}

En el mundo se han desarrollado diferentes estándares para introducir el diseño y aplicación de programas educativos, que permiten valorar el nivel de competencias y la forma que cada persona interactúa en el acceso y uso de la información. En este sentido existen tres estándares que han tenido importancia en el sector educacional:

- Los estándares de la American Library Association and the Association for Educational Communications and Technology (ACRL/ALA, 2002).

- Los estándares australianos para la educación superior (Anziil and Caul Institute, 2004).

- Los estándares de la ALA para la educación superior en el 2000(ALA, 2000).

Otros estándares desarrollados en diferentes universidades son (Castilla, 2008): 
- Nevada Information Literacy Standards, 2002.

- North Dakota Standards and Benchmarks Library/Technology Literacy, 2003.

- Content and Performance Standards for Alaska Students, 2006

- Standards for New Mexico School Libraries, 2001.

\subsection{Programas educativos de Alfabetización informacional}

Con el objetivo de extender las experiencias y los resultados adquiridos en la aplicación de programas educativos de ALFIN, se incrementa en diferentes países el número de organizaciones que se suman al desarrollo de programas que apoyan su objeto de estudio.

Los Estados Unidos es uno de los países precursores en el desarrollo este tipo de programas y los incorpora fundamentalmente dentro de programas de estudios de instituciones y organizaciones educativas (Castilla, 2008):

- Programa Austin, universidad de Texas.

- Programa sobre Nuevas Comunidades de Aprendizaje, Washington, Estados Unidos.

- Programa Tutorial basado en Internet, Minneapolis, Estados Unidos.

- Programa de Olsen y Coons.

- Programa de la Universidad Católica de Australia.

- Programa InfoLit, Región del oeste de África.

- Programa de la Universidad de Kingston, Jamaica.

- Programa de AI y pensamiento crítico, Washington, Estados Unidos.

- Guidelines for Library Media Programs in Louisiana Schools, 2004.

España tiene como alternativa incluir la ALFIN en sus planes de estudio, así como también ocurre en universidades canadienses, francesas y anglosajonas. En países de América Latina como Brasil y Argentina se han desarrollados programas basados en las Tecnologías de la Información y las Comunicaciones (TIC) para la sociedad de la información. Sin embargo el desarrollo de estos programas no han tenido el total apoyo de los gobiernos (Menou, 2004).

\section{ANTECEDENTES DE LA ALFABETIZACIÓN INFORMACIONAL EN CUBA}

La arista de la ALFN que con mayor frecuencia ha sido tratada por la comunidad cubana es el diseño de programas para su ejecución. En este caso los sectores con mayor presencia son la educación superior y la salud pública. A continuación se presentan algunos de los antecedentes de ALFIN en nuestro país mencionados por la MSc. Liuris Rodríguez Castilla:

La ALFIN comenzó en Cuba a principios de la década del 90 desde el Ministerio de Educación Superior, cuando el profesor Lee Tenorio, preparó e impartió cursos de postgrado sobre el empleo del Current Content del Information Sciences Institute utilizando la computación. En sus inicios, los contenidos se centraban en los 
conocimientos sobre cómo realizar búsquedas y revisiones electrónicas, realizar estrategias de búsquedas reusables y adaptables, solicitar artículos a autores, valoración de revistas, factor de impacto y otros índices. A esta temática se le adicionó más tarde, el uso del correo, las búsquedas en Internet, el empleo de los navegadores, las bases de datos, el Web de la Ciencia, los gestores bibliográficos y su aplicación en las investigaciones.

En los años 1996-1997 se incorpora formalmente al curso de Metodología de la Investigación en la Universidad Central de las Villas (UCLV) y en un grupo de especialidades, maestría y doctorados realizados en la Universidad del Distrito de Bogotá, Colombia, entre los años 1996-2000.

En la Facultad de Comunicación de la Universidad de la Habana ha habido un auge en la producción científica desde el 2001, reflejado en las tesis de licenciatura y maestría, y sus implementaciones prácticas a través de cursos auspiciados por la institución.

En el 2004 se realiza un estudio de las potencialidades existentes en la Sede Universitaria Municipal de Plaza de la Revolución que favorecían prácticas de ALFIN en el grupo de estudiantes de trabajadores sociales, logrando como resultado una propuesta de ALFIN.

En el 2005 aparecen dos nuevas aplicaciones, una de ellas fue la propuesta de un programa de ALFIN para los usuarios del Instituto de Neurología y Neurocirugía de La Habana y la otra fue realizada nuevamente en el Centro Nacional de Derecho de Autor.

En el 2006, en la Facultad de Comunicación de la Universidad de La Habana, se analizó el comportamiento informacional en los estudiantes de primer año de las carreras de Periodismo, Comunicación Social, Bibliotecología y Ciencias de la Información. A partir de los resultados obtenidos, se propuso el diseño de un programa de ALFIN. En este mismo año se desarrolla en la Universidad Central de las Villas, un programa de AI a partir de un diagnóstico de su comunidad de usuarios.

\subsection{En la Universidad de las Ciencias Informáticas.}

En el año 2004 el Dr. Lee Tenorio, Director de Informatización del Ministerio de Educación Superior en Cuba, imparte una conferencia que se tituló: "Infotecnología", que poco tiempo después se convirtió en un curso de pregrado y postgrado. Su objetivo fundamental fue demostrar la importancia de la información como recurso para la generación de nuevos conocimientos y el desarrollo científico-investigativo de una universidad. Infotecnología fue solo el nombre, pero su esencia era la ALFIN. 
En el mes de octubre se organiza en el país un Curso-Taller a través del programa PERI11, con la participación de universidades y centros de investigación; dicho programa incluía cuatro componentes en apoyo a la generación de competencias en investigación:

- Suministro de información.

- Diseminación de los resultados de investigaciones nacionales y regionales.

- Fortalecimiento de las habilidades en TIC.

- Fortalecimiento de la publicación local.

Con la conclusión del taller se adquirieron un conjunto de bases de datos que ofrecía el programa, de vital importancia para el desarrollo científico y académico en el país. Todos estos recursos se unen como material imprescindible para los cursos de Infotecnología en la UCI.

A partir de entonces la ALFIN ha dejado de ser un tema desconocido en la universidad, sin embargo no ha sido suficiente para incorporar este indispensable elemento a la práctica efectiva y constante. Si se alfabetiza informacionalmente a los estudiantes entre el tercer y cuarto año se contribuye a garantizar una correcta investigación durante el desarrollo del trabajo de diploma, mediante el desarrollo habilidades y conocimientos para buscar, evaluar, seleccionar, utilizar y comunicar de manera ética la información contenida en documentos y recursos electrónicos. Por estas razones se propone un programa de ALFIN que perfeccione los cursos de pregrado y postgrado utilizados en la UCI actualizándolos con nueva bibliografía e incluyendo nuevos contenidos como la vigilancia tecnológica y el monitoreo de información, principios metodológicos-investigativos, la publicación, presentación en público y socialización de la información de información; dirigido a la integración de dos procesos fundamentales: docencia-investigación.

\section{CONCLUSIONES}

La ALFIN ha cobrado especial interés en la actualidad. Numerosas son las experiencias que existen a nivel internacional. La arista que con mayor frecuencia ha sido tratada por la comunidad cubana es el diseño de programas para su ejecución, sin embargo los mismos no han sido suficientes para que exista una cultura generalizada de las ALFIN por parte de nuestros estudiantes universitarios, los cuales realizan investigaciones bibliográficas para la realización de sus trabajos de diploma y de cualquier otro tipo. Sin embargo en el resto del mundo, sí se manifiestan experiencias enriquecedoras, a las cuales estudiamos e incorporamos en nuestras referencias, como forma de potenciar experiencias y el intercambio.

11PERI: Programme for the Enhancement of Research Information - Programa para el Fortalecimiento de la Información para la Investigación. 


\section{REFERENCIAS}

ACRL/ ALA. (2002). Normas sobre aptitudes para el acceso y uso de la información en la Educación Superior. Asociación Andaluza de Bibliotecarios. [Citado el: 2011 de Mayo de 30.] http://www.aab.es/pdfs/baab60/60a6.pdf. 60.

ALA. (2000)American Library Association. Information Literacy Competency Standards for Higher Education. [Citado el: 30 de Mayo de 2011.] www.ala.org/ala/mgrps/divs/acrl/standards/standards.pdf.

Anziil and Caul InstitutE (2004). Australian and New Zealand Institute for Information Literacy. Australian and New Zealand Information Literacy Framework: principles, standards and practice. [Citado el: 2011 de Mayo de 30.] www.google.com.cu/url?sa=t\&source=web\&cd=2\&ved=0CCAQFjAB\&url=http\%3A \%2F\%2Fciteseerx.ist.psu.edu \%2Fviewdoc\%2Fdownload\%3Fdoi \%3D10.1.1.98.3489\%26 rep $\% 3$ Drep $1 \% 26$ type $\% 3$ Dpdf\&rct=j\&q=Australian $\% 20$ and $\% 20 \mathrm{New} \% 20 \mathrm{Zealand} \% 20 \mathrm{In}$ formation $\% 20$ Literacy $\% 20$ Framework $\% 3 \mathrm{~A} \% 2$.

Bawden, D. (2002). Anales de la Documentación. Revisión de los conceptos de Alfabetización informacional y Alfabetización Digital. [Citado el: 25 de abril de 2011.] http:/ / redalyc.uaemex.mx/src/inicio/ArtPdfRed.jsp?iCve=63500518. 005.

Bruce, C. S. (2003). Anales de la Documentación. Las siete caras de la Alfabetización en Información en la Enseñanza Superior. [Citado el: 25 de abril de 2011.] http://redalyc.uaemex.mx/pdf/635/63500619.pdf. 006.

Castilla, L. (2008). Ciencias de la Información. La Alfabetización Informacional en los procesos de desarrollo de software. Propuesta de un programa para la Universidad de las Ciencias Informáticas. [Citado el: 20 de Mayo de 2011.]

http://cinfo.idict.cu/index.php/cinfo/article/view/81/74. 3.

Council of Australian University Librarians (2002). Asociación andaluza de bibliotecarios. Normas sobre alfabetización en información. [Citado el: 23 de Marzo de 2011.] www.aab.es/pdfs/baab68/68a4.pdf. 68.

Menou, M. J. (2004). Redalyc. La alfabetización informacional dentro de las politicas nacionales sobre tecnologías de la información y comunicación (tics): la cultura de la información, una dimensión ausente. [Citado el: 20 de Mayo de 2011.] http://redalyc.uaemex.mx/redalyc/src/inicio/ArtPdfRed.jsp?iCve=63500716.

Pinto, M. y García, J. (2006). Espacio Europeo para la Educación Superior. La enseñanza-aprendizaje de las competencias genéricas en el Espacio Europeo de Educación Superior: el proyecto ALFINEES. [Citado el: 26 de abril de 2011.] www.unizar.es/eees/innovacion06/COMUNIC_PUBLI/BLOQUE_II/CAP_II_9.pdf. 
Ruíz, E. (2009). Revista Digital Sociedad de la Información. LA ALFABETIZACIÓN INFORMACIONAL. [Citado el: 06 de junio de 2011.] www.sociedadelainformacion.com/16/alfabetizacion.pdf.

Sáez, E. (2005). Gestiopolis. Alfabetización en información y participación ciudadana. [Citado el: 23 de Marzo de 2011.] http://www.gestiopolis.com/recursos5/docs/eco/alfaben.htm.

\section{Isabel González Flores}

Graduada en Ingeniería en Ciencias de la Computación por la Universidad de las Ciencias Informáticas de la Habana, en Cuba. Actualmente ejerce en el Centro de Gobierno Electrónico (CEGEL), Facultad 3, de dicha Universidad y participa en proyectos de la institución. También ha sido miembro activo en la organización y participación de eventos académicos, nacionales e internacionales, además de haber publicado en revistas y memorias de congresos y talleres. 